\title{
The Ptolemy field of 3-manifold representations
}

\author{
STAVROS GAROUFALIDIS \\ MATTHIAS GOERNER \\ CHRISTIAN K ZICKERT
}

\begin{abstract}
The Ptolemy coordinates for boundary-unipotent $\operatorname{SL}(n, \mathbb{C})$-representations of a 3manifold group were introduced by Garoufalidis, Thurston and Zickert [10] inspired by the $\mathcal{A}$-coordinates on higher Teichmüller space due to Fock and Goncharov. We define the Ptolemy field of a (generic) PSL(2, $\mathbb{C}$ )-representation and prove that it coincides with the trace field of the representation. This gives an efficient algorithm to compute the trace field of a cusped hyperbolic manifold.
\end{abstract}

$57 \mathrm{~N} 10 ; 57 \mathrm{M} 27$

\section{Introduction}

\subsection{The Ptolemy coordinates}

The Ptolemy coordinates for boundary-unipotent representations of a 3-manifold group in $\operatorname{SL}(n, \mathbb{C})$ were introduced by Garoufalidis, Thurston and Zickert [10], inspired by the $\mathcal{A}$-coordinates on higher Teichmüller space due to Fock and Goncharov [7]. In this paper we will focus primarily on representations in $\operatorname{SL}(2, \mathbb{C})$ and $\operatorname{PSL}(2, \mathbb{C})$.

Given a topological ideal triangulation $\mathcal{T}$ of an oriented compact 3-manifold $M$, a Ptolemy assignment (for $\mathrm{SL}(2, \mathbb{C})$ ) is an assignment of a non-zero complex number (called a Ptolemy coordinate) to each $1-$ cell of $\mathcal{T}$ such that, for each simplex, the Ptolemy coordinates assigned to the edges $\varepsilon_{i j}$ satisfy the Ptolemy relation

$$
c_{03} c_{12}+c_{01} c_{23}=c_{02} c_{13} .
$$

The set of Ptolemy assignments is thus an affine variety $P_{2}(\mathcal{T})$, which is cut out by homogeneous quadratic polynomials.

We define the Ptolemy field of a boundary-unipotent representation and show that it is isomorphic to the trace field. This gives rise to an efficient algorithm for exact computation of the trace field of a hyperbolic manifold. 


\subsection{Decorated SL $(2, \mathbb{C})$-representations}

The precise relationship between Ptolemy assignments and representations is given by (1-2) $\left\{\right.$ Points in $\left.P_{2}(\mathcal{T})\right\} \stackrel{1-1}{\longleftrightarrow}\{$ Natural $(\operatorname{SL}(2, \mathbb{C}), P)$-cocycles on $M\}$

$$
\stackrel{1-1}{\longleftrightarrow}\{\text { Generically decorated }(\mathrm{SL}(2, \mathbb{C}), P) \text {-representations }\} .
$$

The concepts are briefly described below, and the correspondences are illustrated in the right image in Figure 3 and in Figure 2. We refer to Section 2 for a summary of our notation. The bijections of (1-2) first appeared in Zickert [14] (in a slightly different form), and were generalized to $\operatorname{SL}(n, \mathbb{C})$-representations by Garoufalidis, Thurston and Zickert [10].

- Natural cocycle Labeling of the edges of each truncated simplex by elements in $\operatorname{SL}(2, \mathbb{C})$ satisfying the cocycle condition (the product around each face is $1)$. The long edges are counter-diagonal, ie of the form

$$
\left(\begin{array}{cc}
0 & -x^{-1} \\
x & 0
\end{array}\right)
$$

and the short edges are nontrivial elements in $P$. Identified edges are labeled by the same group element.

- Decorated representation A decoration of a boundary-parabolic representation $\rho$ is an assignment of a coset $g P$ to each vertex of $\widehat{\vec{M}}$ which is equivariant with respect to $\rho$. A decoration is generic if for each edge joining two vertices, the two $P$-cosets $g P, h P$ are distinct as $B$-cosets. This condition is equivalent to $\operatorname{det}\left(g e_{1}, h e_{1}\right) \neq 0$. Two decorations are considered equal if they differ by left multiplication by a group element $g$.
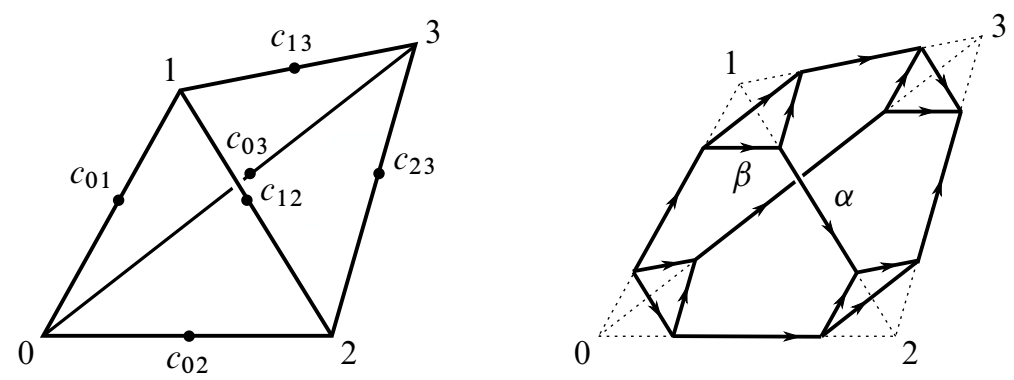

Figure 1: Left: Ptolemy assignment; the Ptolemy relation (1-1) holds. Right: natural cocycle; $\alpha$ is counter-diagonal, $\beta \in P$.

By ignoring the decoration, (1-2) yields a map

$$
\mathcal{R}: P_{2}(\mathcal{T}) \rightarrow\{(\operatorname{SL}(2, \mathbb{C}), P) \text {-representations }\} / \text { Conj }
$$




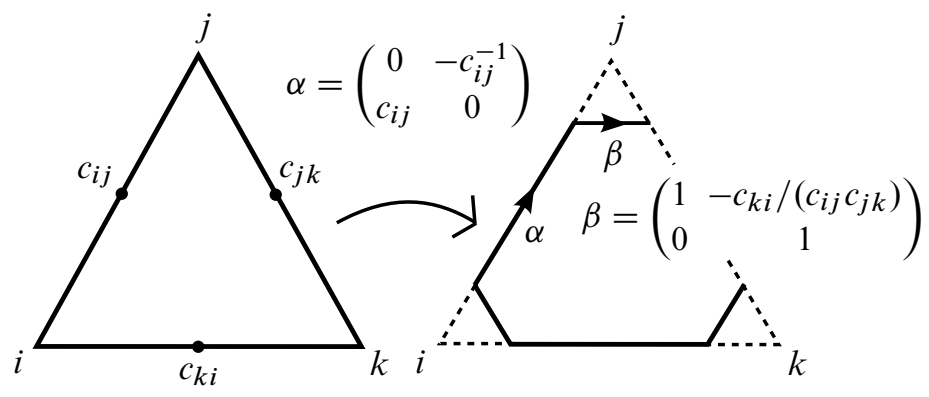

Figure 2: From Ptolemy assignments to natural cocycles

The representation corresponding to a Ptolemy assignment is given explicitly in terms of the natural cocycle.

Remark 1.1 Note that a natural cocycle canonically determines a representation of the edge path groupoid of the triangulation of $M$ by truncated simplices.

Remark 1.2 A decoration of $\rho$ determines a developing map $\widehat{\widehat{M}} \rightarrow \overline{\mathbb{H}}^{3}$ by straightening the simplices. We shall not need this here. For a discussion of the relationship between decorations and developing maps, see Zickert [14]. For general theory of developing maps, see Dunfield [4].

Remark 1.3 Every boundary-parabolic representation has a decoration, but a representation may have only non-generic decorations. The map $\mathcal{R}$ is thus not surjective in general, and the image depends on the triangulation. However, if the triangulation is sufficiently fine, $\mathcal{R}$ is surjective (see Garoufalidis, Thurston and Zickert [10]). The preimage of a representation depends on the image of the peripheral subgroups (see Proposition 1.10).
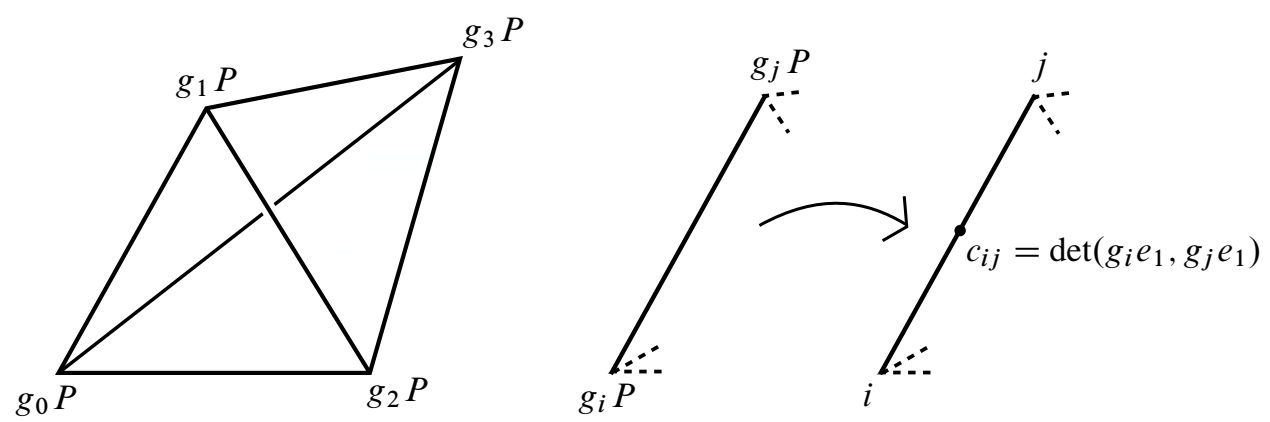

Figure 3: Left: decoration; equivariant assignment of cosets. Right: from decorations to Ptolemy assignments. 


\subsection{Obstruction classes and PSL $(2, \mathbb{C})$-representations}

There is a subtle distinction between representations in $\operatorname{SL}(2, \mathbb{C})$ versus $\operatorname{PSL}(2, \mathbb{C})$. The geometric representation of a hyperbolic manifold always lifts to an $\operatorname{SL}(2, \mathbb{C})$ representation, but for a one-cusped manifold, no lift is boundary-parabolic (any lift will take a longitude to an element of trace -2 ; see Calegari [2]).

The obstruction to lifting a boundary-parabolic $\operatorname{PSL}(2, \mathbb{C})$-representation to a boundaryparabolic $\operatorname{SL}(2, \mathbb{C})$-representation is a class in $H^{2}(\widehat{M} ; \mathbb{Z} / 2 \mathbb{Z})$. For each such class, there is a Ptolemy variety $P_{2}^{\sigma}(\mathcal{T})$, which maps to the set of PSL $(2, \mathbb{C})$-representations with obstruction class $\sigma$. More precisely, $P_{2}^{\sigma}(\mathcal{T})$ is defined for each 2-cocycle $\sigma \in Z^{2}(\widehat{M} ; \mathbb{Z} / 2 \mathbb{Z})$, and up to canonical isomorphism only depends on the cohomology class of $\sigma$. The Ptolemy variety for the trivial cocycle equals $P_{2}(\mathcal{T})$. The analogue of $(1-2)$ is

$$
\text { \{Points in } \begin{aligned}
\left.P_{2}^{\sigma}(\mathcal{T})\right\} & \stackrel{1-1}{\longrightarrow}\left\{\begin{array}{c}
\text { Lifted natural }(\operatorname{SL}(2, \mathbb{C}), P)-\text { cocycles } \\
\text { with obstruction cocycle } \sigma
\end{array}\right\} \\
& \longrightarrow\left\{\begin{array}{c}
\text { Generically decorated }(\operatorname{SL}(2, \mathbb{C}), P)- \\
\text { representations with obstruction class } \sigma
\end{array}\right\} .
\end{aligned}
$$

A lifted natural cocycle is defined as above, except that the product along a face is now $\pm I$, where the sign is determined by $\sigma$. The right map is no longer a $1-1$ correspondence; the preimage of each decorated representation is the choice of lifts, ie parametrized by a cocycle in $Z^{1}(\widehat{M} ; \mathbb{Z} / 2 \mathbb{Z})$. We refer to [10] for details. As in (1-3), ignoring the decoration yields a map

$$
\mathcal{R}: P_{2}^{\sigma}(\mathcal{T}) \rightarrow\left\{\begin{array}{c}
(\operatorname{PSL}(2, \mathbb{C}), P) \text {-representations } \\
\text { with obstruction class } \sigma
\end{array}\right\} / \text { Conj }
$$

which is explicitly given in terms of the natural cocycle.

Theorem 1.4 (Garoufalidis, Thurston and Zickert [10]) If $M$ is hyperbolic, and all edges of $\mathcal{T}$ are essential, the geometric representation is in the image of $\mathcal{R}$.

Remark 1.5 If $\mathcal{T}$ has a non-essential edge, all Ptolemy varieties will be empty. Hence, if $P_{2}^{\sigma}(\mathcal{T})$ is non-empty for some $\sigma$, and if $M$ is hyperbolic, the geometric representation is detected by the Ptolemy variety of the geometric obstruction class.

\subsection{Our results}

We view the Ptolemy varieties $P_{2}^{\sigma}(\mathcal{T})$ as subsets of an ambient space $\mathbb{C}^{e}$, with coordinates indexed by the 1 -cells of $\mathcal{T}$. Let $T=\left(\mathbb{C}^{*}\right)^{v}$, with the coordinates indexed by the boundary components of $M$. 
Definition 1.6 The diagonal action is the action of $T$ on $P_{2}^{\sigma}(\mathcal{T})$, where an element $\left(x_{1}, \ldots, x_{v}\right) \in T$ acts on a Ptolemy assignment by replacing the Ptolemy coordinate $c$ of an edge $e$ with $x_{i} x_{j} c$, where $x_{i}$ and $x_{j}$ are the coordinates corresponding to the ends of $e$. Let

$$
P_{2}^{\sigma}(\mathcal{T})_{\text {red }}=P_{2}^{\sigma}(\mathcal{T}) / T
$$

Definition 1.7 A boundary-parabolic $\operatorname{PSL}(2, \mathbb{C})$-representation is generic if it has a generic decoration. It is boundary-nontrivial if each peripheral subgroup has nontrivial image.

Remark 1.8 Note that the notion of genericity is with respect to the triangulation. By Theorem 1.4, if all edges of $\mathcal{T}$ are essential (and $\mathcal{T}$ has no interior vertices), the geometric representation of a cusped hyperbolic manifold is always generic and boundary-nontrivial.

Remark 1.9 Note that if $M$ has spherical boundary components (eg if $\mathcal{T}$ is a triangulation of a closed manifold), no representation is boundary-nontrivial.

Proposition 1.10 The map $\mathcal{R}$ in (1-5) factors through $P_{2}^{\sigma}(\mathcal{T})_{\text {red }}$, ie we have

$$
\mathcal{R}: P_{2}^{\sigma}(\mathcal{T})_{\text {red }} \rightarrow\left\{\begin{array}{c}
(\operatorname{PSL}(2, \mathbb{C}), P) \text {-representations } \\
\text { with obstruction class } \sigma
\end{array}\right\} / \text { Conj. }
$$

The image is the set of generic representations, and the preimage of a generic, boundarynontrivial representation is finite and parametrized by $H^{1}(\widehat{M} ; \mathbb{Z} / 2 \mathbb{Z})$.

Remark 1.11 For the corresponding map from $P_{2}(\mathcal{T})_{\text {red }}$ to $(\operatorname{SL}(2, \mathbb{C}), P)$-representations, the preimage of a generic boundary-nontrivial representation is a single point.

Remark 1.12 The preimage of a representation which is not boundary-nontrivial is never finite. In fact, its dimension is the number of boundary components that are collapsed. In particular, it follows that if $c \in P_{2}^{\sigma}(\mathcal{T})_{\text {red }}$ is in a 0 -dimensional component (which is not contained in a higher-dimensional component), the image is boundary-nontrivial.

By geometric invariant theory, $P_{2}^{\sigma}(\mathcal{T})_{\text {red }}$ is a variety whose coordinate ring is the ring of invariants $\mathcal{O}^{T}$ of the coordinate ring $\mathcal{O}$ of $P_{2}^{\sigma}(T)$.

Definition 1.13 Let $c \in P_{2}^{\sigma}(\mathcal{T})$. The Ptolemy field of $c$ is the field

$$
k_{c}=\mathbb{Q}\left(\left\{p\left(c_{1}, \ldots, c_{e}\right) \mid p \in \mathcal{O}^{T}\right\}\right) .
$$

The Ptolemy field of a generic boundary-nontrivial representation is the Ptolemy field of any preimage under (1-7). 
Clearly, the Ptolemy field only depends on the image in $P_{2}^{\sigma}(\mathcal{T})_{\text {red }}$. Our main result is the following.

Theorem 1.14 The Ptolemy field of a boundary-nontrivial, generic, boundary-parabolic representation $\rho$ in $\operatorname{PSL}(2, \mathbb{C})$ or $\operatorname{SL}(2, \mathbb{C})$ is equal to its trace field.

Remark 1.15 For a cusped hyperbolic 3-manifold the shape field is in general smaller than the trace field. The shape field equals the invariant trace field (see eg Maclachlan and Reid [12]).

For computations of the Ptolemy field, we need an explicit description of the ring of invariants $\mathcal{O}^{T}$, or, equivalently, the reduced Ptolemy variety $P_{2}^{\sigma}(\mathcal{T})_{\text {red }}$.

Proposition 1.16 There exist 1 -cells $\varepsilon_{1}, \ldots, \varepsilon_{v}$ of $\mathcal{T}$ such that the reduced Ptolemy variety $P_{2}^{\sigma}(\mathcal{T})_{\text {red }}$ is naturally isomorphic to the subvariety of $P_{2}^{\sigma}(\mathcal{T})$ obtained by intersecting with the affine hyperplane $c_{\varepsilon_{1}}=\cdots=c_{\varepsilon_{v}}=1$.

Corollary 1.17 Let $c \in P_{2}^{\sigma}(\mathcal{T})_{\text {red }}$. Under an isomorphism as in Proposition 1.16, the Ptolemy field of $c$ is the field generated by the Ptolemy coordinates.

Remark 1.18 A concrete method for selecting 1-cells as in Proposition 1.16 is described in Section 4.3.

Analogues of our results for higher-rank Ptolemy varieties are discussed in Section 6. The analogue of Proposition 1.10 holds for representations that are boundary-nondegenerate (see Definition 6.10), and the analogue of Proposition 1.16 leads to a simple computation of the Ptolemy field.

Conjecture 1.19 The Ptolemy field of a boundary-non-degenerate, generic, boundaryunipotent representation $\rho$ in $\operatorname{SL}(n, \mathbb{C})$ or $\operatorname{PSL}(n, \mathbb{C})$ is equal to its trace field.

Remark 1.20 The computation of reduced Ptolemy varieties is remarkably efficient using Magma [1]. For all but a few census manifolds, primary decompositions of the (reduced) Ptolemy varieties $P_{2}^{\sigma}(\mathcal{T})$ can be computed in a fraction of a second on a standard laptop. A database can be found at CURVE [5]; see also Falbel, Koseleff and Rouillier [6]. All of our tools have been incorporated into SnapPy [3] by the second author and the Ptolemy fields can be obtained through the command below:

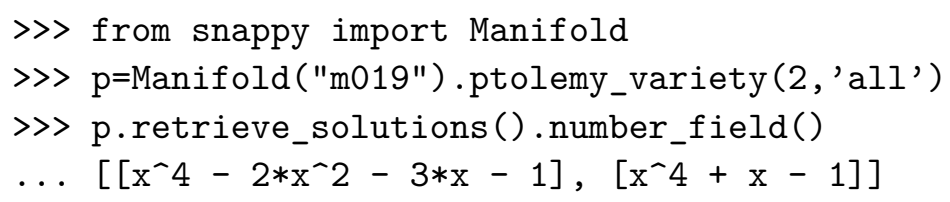


The number fields are grouped by obstruction class. In this example, we see that the Ptolemy variety for the nontrivial obstruction class has a component with number field $x^{4}+x-1$, which is the trace field of m019. The above code retrieves a precomputed decomposition of the Ptolemy variety from CURVE [5]. In Sage or SnapPy with Magma installed, you can use p.compute_solutions ().number_field() to compute the decomposition.

Acknowledgements Stavros Garoufalidis and Christian Zickert were supported in part by NSF grants number DMS-14-06419 and DMS-13-09088, respectively.

\section{Notation}

\subsection{Triangulations}

Let $M$ be a compact oriented 3-manifold with (possibly empty) boundary. We refer to the boundary components as cusps (although they may not be tori). Let $\tilde{M}$ be the universal cover of $M$ and let $\widehat{M}$ and $\widehat{M}$, respectively, be the spaces obtained from $M$ and $\tilde{M}$ by collapsing each boundary component to a point.

Definition 2.1 A (concrete) triangulation of $M$ is an identification of $\widehat{M}$ with a space obtained from a collection of simplices by gluing together pairs of faces by affine homeomorphisms. For each simplex $\Delta$ of $\mathcal{T}$ we fix an identification of $\Delta$ with a standard simplex.

Remark 2.2 By drilling out disjoint balls if necessary (this does not change the fundamental group), we may assume that the triangulation of $M$ is ideal, ie that each 0 -cell corresponds to a boundary component of $M$. For example, we regard a triangulation of a closed manifold as an ideal triangulation of a manifold with boundary a union of spheres.

Definition 2.3 A triangulation is oriented if the identifications with standard simplices are orientation-preserving.

Remark 2.4 The triangulations in the SnapPy censuses OrientableCuspedCensus, LinkExteriors and HTLinkExteriors [3] are oriented. Unless otherwise specified we shall assume that our triangulations are oriented.

A triangulation gives rise to a triangulation of $M$ by truncated simplices, and to a triangulation of $\widehat{\widehat{M}}$. 


\subsection{Miscellaneous}

- The number of vertices, edges, faces and simplices, of a triangulation $\mathcal{T}$ are denoted by $v, e, f$ and $s$, respectively.

- The standard basis vectors in $\mathbb{Z}^{k}$ are denoted by $e_{1}, \ldots, e_{k}$.

- The (oriented) edge of simplex $k$ from vertex $i$ to $j$ is denoted by $\varepsilon_{i j, k}$.

- The matrix groups $\left\{\left(\begin{array}{ll}1 & x \\ 0 & 1\end{array}\right)\right\}$ and $\left\{\left(\begin{array}{ll}a & x \\ 0 & a^{-1}\end{array}\right)\right\}$ are denoted by $P$ and $B$, respectively. The higher-rank analogue of $P$ is denoted by $N$.

- A representation is boundary-parabolic if it takes each peripheral subgroup to a conjugate of $P$. Such is also called a $(G, P)$-representation $(G=\mathrm{SL}(2, \mathbb{C})$ or $\operatorname{PSL}(2, \mathbb{C}))$. In the higher-rank case, such a representation is called boundaryunipotent.

- A triangulation is ordered if $\varepsilon_{i j, k} \sim \varepsilon_{i^{\prime} j^{\prime}, k^{\prime}}$ implies that $i<j \Longleftrightarrow i^{\prime}<j^{\prime}$.

\section{The Ptolemy varieties}

We define the Ptolemy variety for $n=2$ following Garoufalidis, Thurston and Zickert [10] (see also Garoufalidis, Goerner and Zickert [8]).

\subsection{The SL $(2, \mathbb{C})$-Ptolemy variety}

Assign to each oriented edge $\varepsilon_{i j, k}$ of $\Delta_{k} \in \mathcal{T}$ a Ptolemy coordinate $c_{i j, k}$. Consider the affine algebraic set $A$ defined by the Ptolemy relations

$$
c_{03, k} c_{12, k}+c_{01, k} c_{23, k}=c_{02, k} c_{13, k}, \quad k=1,2, \ldots, t,
$$

the identification relations

$$
c_{i j, k}=c_{i^{\prime} j^{\prime}, k^{\prime}} \quad \text { when } \quad \varepsilon_{i j, k} \sim \varepsilon_{i^{\prime} j^{\prime}, k^{\prime}},
$$

and the edge orientation relations $c_{i j, k}=-c_{j i, k}$. By only considering $i<j$, we shall always eliminate the edge orientation relations.

Definition 3.1 The Ptolemy variety $P_{2}(\mathcal{T})$ is the Zariski open subset of $A$ consisting of points with non-zero Ptolemy coordinates.

Remark 3.2 One can concretely obtain $P_{2}(\mathcal{T})$ from $A$ by adding a dummy variable $x$ and a dummy relation $x \cdot \prod c_{i j, k}=1$.

Remark 3.3 We can eliminate the identification relations (3-2) by selecting a representative for each edge cycle. This gives an embedding of the Ptolemy variety in an ambient space $\mathbb{C}^{e}$, where it is cut out by $s$ Ptolemy relations, one for each simplex. Note that when all boundary components are tori, $s=e$. 
3.1.1 The figure-8 knot Consider the ideal triangulation of the figure- 8 knot complement shown in Figure 4. The Ptolemy variety $P_{2}(\mathcal{T})$ is given by

$$
\begin{aligned}
& c_{03,0} c_{12,0}+c_{01,0} c_{23,0}=c_{02,0} c_{13,0}, \\
& c_{03,1} c_{12,1}+c_{01,1} c_{23,1}=c_{02,1} c_{13,1}, \\
& c_{02,0}=c_{12,0}=c_{13,0}=c_{01,1}=c_{03,1}=c_{23,1}, \\
& c_{01,0}=c_{03,0}=c_{23,0}=c_{02,1}=c_{12,1}=c_{13,1} .
\end{aligned}
$$

By selecting representatives $\varepsilon_{23,0}$ and $\varepsilon_{13,0}$ for the two edge cycles, $P_{2}(\mathcal{T})$ embeds in $\mathbb{C}^{2}$, where it is given by

$$
c_{23,0} c_{13,0}+c_{23,0}^{2}=c_{13,0}^{2}, \quad c_{13,0} c_{23,0}+c_{13,0}^{2}=c_{23,0}^{2} .
$$

It follows that $P_{2}(\mathcal{T})$ is empty, which is no surprise, since the only boundary-parabolic $\mathrm{SL}(2, \mathbb{C})$-representations of the figure- 8 knot are abelian. To detect the geometric representation, we need to consider obstruction classes (see Section 3.2 below).

3.1.2 The figure-8 knot sister Consider the ideal triangulation of the figure- 8 knot sister shown in Figure 5. The Ptolemy variety $P_{2}(\mathcal{T})$ is given by

$$
\begin{aligned}
& c_{03,0} c_{12,0}+c_{01,0} c_{23,0}=c_{02,0} c_{13,0}, \\
& c_{03,1} c_{12,1}+c_{01,1} c_{23,1}=c_{02,1} c_{13,1}, \\
& c_{01,0}=-c_{03,0}=c_{23,0}=-c_{01,1}=c_{03,1}=-c_{23,1}, \\
& c_{02,0}=-c_{12,0}=c_{13,0}=-c_{02,1}=c_{12,1}=-c_{13,1} .
\end{aligned}
$$

Selecting representatives $\varepsilon_{23,0}$ and $\varepsilon_{13,0}$ for the two edge cycles, $P_{2}(\mathcal{T}) \in \mathbb{C}^{2}$ is given by

$$
c_{23,0} c_{13,0}+c_{23,0}^{2}=c_{13,0}^{2}, \quad c_{23,0} c_{13,0}+c_{23,0}^{2}=c_{13,0}^{2} .
$$

This is equivalent to

$$
x^{2}-x-1=0, \quad x=\frac{c_{13,0}}{c_{23,0}} .
$$

Remark 3.4 Note that, for ordered triangulations, the identification relations (3-2) do not involve minus signs. The triangulation in Figure 4 is not oriented.

\subsection{Obstruction classes}

Each class in $H^{2}(\widehat{M} ; \mathbb{Z} / 2 \mathbb{Z})$ can be represented by a $\mathbb{Z} / 2 \mathbb{Z}$-valued 2-cocycle on $\widehat{M}$, ie an assignment of a sign to each face of $\mathcal{T}$. 


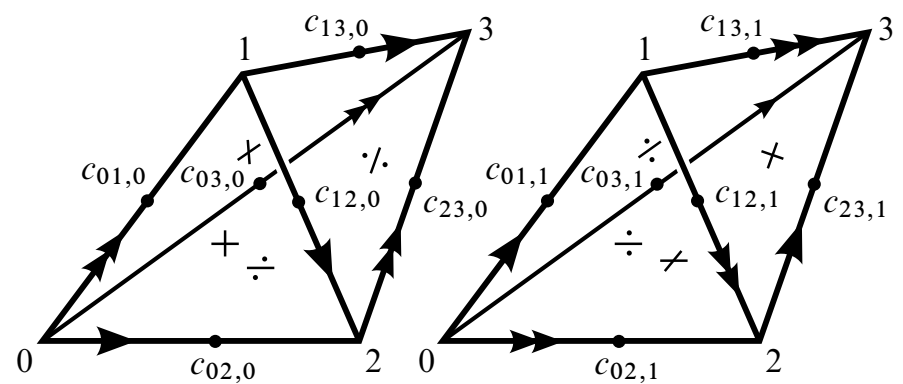

Figure 4: Ordered triangulation of the figure- 8 knot. The signs indicate the nontrivial obstruction class.

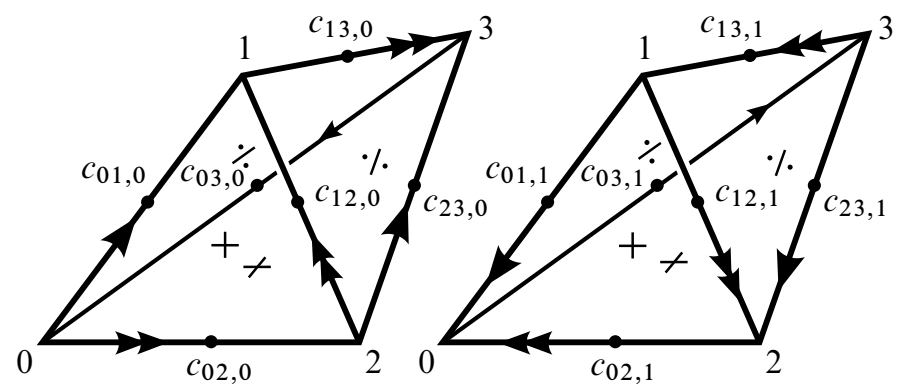

Figure 5: Oriented triangulation of the figure- 8 knot sister. The signs indicate the nontrivial obstruction class.

Definition 3.5 Let $\sigma$ be a $\mathbb{Z} / 2 \mathbb{Z}$-valued 2-cocycle on $\widehat{M}$. The Ptolemy variety for $\sigma$ is defined as in Definition 3.1, but with the Ptolemy relation replaced by

$$
\sigma_{0, k} \sigma_{3, k} c_{03, k} c_{12, k}+\sigma_{0, k} \sigma_{1, k} c_{01, k} c_{23, k}=\sigma_{0, k} \sigma_{2, k} c_{02, k} c_{13, k},
$$

where $\sigma_{i, k}$ is the sign of the face of $\Delta_{k}$ opposite vertex $i$.

Remark 3.6 Multiplying $\sigma$ by a coboundary $\delta(\tau)$ corresponds to multiplying the Ptolemy coordinate of a one-cell $e$ by $\tau(e)$ (see [10] for details). Hence, up to canonical isomorphism, the Ptolemy variety $P_{2}^{\sigma}(\mathcal{T})$ only depends on the cohomology class of $\sigma$. The Ptolemy variety $P_{2}(\mathcal{T})$ is the Ptolemy variety for the trivial obstruction class.

3.2.1 Examples In both examples above, $H^{2}(\widehat{M} ; \mathbb{Z} / 2 \mathbb{Z})=\mathbb{Z} / 2 \mathbb{Z}$, and the nontrivial obstruction class $\sigma$ is indicated in Figures 4 and 5 .

For the figure- 8 knot, $P_{2}^{\sigma}(\mathcal{T})$ is given by

$$
-c_{23,0} c_{13,0}+c_{23,0}^{2}=-c_{13,0}^{2}, \quad-c_{13,0} c_{23,0}+c_{13,0}^{2}=-c_{23,0}^{2},
$$


which is equivalent to

$$
x^{2}-x+1=0, \quad x=\frac{c_{13,0}}{c_{23,0}} \text {. }
$$

The corresponding representations are the geometric representation and its conjugate.

For the figure- 8 knot sister, the Ptolemy variety becomes

$$
-c_{23,0} c_{13,0}-c_{23,0}^{2}=c_{13,0}^{2}, \quad-c_{23,0} c_{13,0}-c_{23,0}^{2}=c_{13,0}^{2},
$$

which is equivalent to

$$
x^{2}+x+1=0, \quad x=\frac{c_{13,0}}{c_{23,0}} .
$$

\section{The diagonal action}

Fix an ordering of the 1-cells of $\mathcal{T}$ and of the cusps of $M$. As mentioned in Remark 3.3, the Ptolemy variety can be regarded as a subset of the ambient space $\mathbb{C}^{e}$.

Let $T=\left(\mathbb{C}^{*}\right)^{v}$ be a torus whose coordinates are indexed by the cusps of $M$. There is a natural action of $T$ on $P_{2}^{\sigma}(\mathcal{T})$ defined as follows: for $x=\left(x_{1}, \ldots, x_{v}\right) \in T$ and $c=\left(c_{1}, \ldots c_{e}\right) \in P_{2}^{\sigma}(\mathcal{T})$, define a Ptolemy assignment $c x$ by

$$
(x c)_{i}=x_{j} x_{k} c_{i}
$$

where $j$ and $k$ (possibly $j=k$ ) are the cusps joined by the $i^{\text {th }}$ edge cycle. The action is thus determined entirely by the $1-$ skeleton of $\widehat{M}$.

Remark 4.1 There is a more intrinsic definition of this action in terms of decorations: Each vertex of $\widehat{M}$ determines a cusp of $M$, and if $D$ is a decoration taking a vertex $w$ to $g P$, the decoration $x D$ takes $w$ to

$$
g\left(\begin{array}{cc}
x_{i} & 0 \\
0 & x_{i}^{-1}
\end{array}\right) P
$$

where $i$ is the cusp determined by $w$. The fact that the two definitions agree under the one-to-one correspondence (1-4) is an immediate consequence of the relationship given in the right image in Figure 3. 


\subsection{The reduced Ptolemy varieties}

Definition 4.2 The reduced Ptolemy variety $P_{2}^{\sigma}(\mathcal{T})_{\text {red }}$ is the quotient $P_{2}^{\sigma}(\mathcal{T}) / T$.

Let $\mathcal{O}$ be the coordinate ring of $P_{2}^{\sigma}(\mathcal{T})$, and let $\mathcal{O}^{T}$ be the ring of invariants. By geometric invariant theory, the reduced Ptolemy variety is a variety whose coordinate ring is isomorphic to $\mathcal{O}^{T}$.

For $i=0,1$, let $C_{i}$ denote the free abelian group generated by the unoriented $i$-cells of $\widehat{M}$, and consider the maps (first studied by Neumann [13])

$$
\alpha: C_{0} \rightarrow C_{1}, \quad \alpha^{*}: C_{1} \rightarrow C_{0},
$$

where $\alpha$ takes a 0 -cell to the sum of its incident 1 -cells, and $\alpha^{*}$ takes a 1 -cell to the sum of its endpoints. The maps $\alpha$ and $\alpha^{*}$ are dual under the canonical identifications $C_{i} \cong C_{i}^{*}$. Also, $\alpha$ is injective, and $\alpha^{*}$ has cokernel of order 2 (see [13]).

The following is an elementary consequence of the definition of the diagonal action.

Lemma 4.3 The diagonal action $P_{2}^{\sigma}(\mathcal{T})$ and the induced action on the coordinate ring $\mathcal{O}$ of $P_{2}^{\sigma}(\mathcal{T})$ are given, respectively, by

$$
(x c)_{i}=\left(\prod_{j=1}^{v} x_{j}^{\alpha_{i j}}\right) c_{i}, \quad x\left(c^{w}\right)=\prod_{j=1}^{v} x_{j}^{\alpha^{*}(w)_{j}} c^{w},
$$

where $c^{w}$ is the monomial $c_{1}^{w_{1}} \cdots c_{e}^{w_{e}} \in \mathcal{O}, w \in \mathbb{Z}^{e}$.

Corollary 4.4 Suppose that $w_{1}, \ldots, w_{e-v}$ form a basis for $\operatorname{Ker} \alpha^{*}$. The monomials $c^{w_{1}}, \ldots, c^{w_{e-v}}$ generate $\mathcal{O}^{T}$.

4.1.1 Examples Suppose the 1-skeleton of $\widehat{M}$ looks like the left image in Figure 6 (this is in fact the 1-skeleton of the census triangulation of the Whitehead link complement). We have

$$
\alpha^{*}=\left(\begin{array}{llll}
2 & 1 & 1 & 0 \\
0 & 1 & 1 & 2
\end{array}\right)
$$

and the action of $\left(x_{1}, x_{2}\right)$ on a Ptolemy assignment $c$ is given in the right image in Figure 6.

The kernel of $\alpha^{*}$ is generated by $(0,-2,0,1)^{t}$ and $(-1,1,1,0)^{t}$, so we have

$$
\mathcal{O}^{T}=\left\langle c_{2}^{-2} c_{4}, c_{1}^{-1} c_{2} c_{3}\right\rangle \text {. }
$$

Also note that, in each of the examples in Section 3, $x \in \mathcal{O}^{T}$.

For computations we need a more explicit description of the reduced Ptolemy variety. 

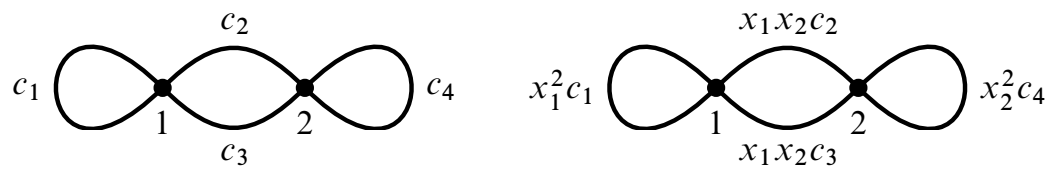

Figure 6: Left: Ptolemy assignment. Right: the diagonal action of $\left(x_{1}, x_{2}\right)$.

Definition 4.5 Let $T: \mathbb{Z}^{n} \rightarrow \mathbb{Z}^{m}$ be a homomorphism. We say that $T$ is basic if there exists a subset $J$ of $\left\{e_{1}, \ldots, e_{n}\right\}$ such that $T$ maps $\operatorname{Span}(J)$ isomorphically onto the image of $T$. Elements of such a set $J$ are called basic generators for $T$.

We identify $C_{1}$ and $C_{0}$ with $\mathbb{Z}^{e}$ and $\mathbb{Z}^{v}$, respectively.

Proposition 4.6 The map $\alpha^{*}: C_{1} \rightarrow C_{0}$ is basic.

The proof will be relegated to Section 4.3, where we shall also give explicit basic generators.

Proposition 4.7 Let $\varepsilon_{i_{1}}, \ldots, \varepsilon_{i_{v}}$ be basic generators for $\alpha^{*}$. The ring of invariants $\mathcal{O}^{T}$ is isomorphic to $\mathbb{C}\left[c_{1}, \ldots, c_{e}\right]$ modulo the Ptolemy relations and the relations $c_{i_{1}}=$ $\cdots=c_{i_{v}}=1$, ie the reduced Ptolemy variety is isomorphic to the subset of $P_{2}^{\sigma}(\mathcal{T})$ where the Ptolemy coordinates of the basic generators are 1.

Proof Let $w_{1}, \ldots, w_{e-v}$ be a basis for Ker $\alpha^{*}$. Hence, $w_{1}, \ldots, w_{e-v}$ and $\varepsilon_{i_{1}}, \ldots, \varepsilon_{i_{v}}$ generate $C_{1}$. We can thus uniquely express each $c_{i}$ as a monomial in the $w_{j}$ and the $c_{i_{j}}$. The result now follows from Corollary 4.4 .

Remark 4.8 This is how the Ptolemy varieties are computed in SnapPy.

\subsection{Shapes and gluing equations}

One can assign to each simplex a shape

$$
z=\sigma_{3} \sigma_{2} \frac{c_{03} c_{12}}{c_{02} c_{13}} \in \mathbb{C} \backslash\{0,1\},
$$

and one can show (see $[10 ; 8]$ ) that these satisfy Thurston's gluing equations. For the geometric representation of a cusped hyperbolic manifold, the shape field (field generated by the shapes) is equal to the invariant trace field, which is in general smaller than the trace field; see Maclachlan and Reid [12].

Remark 4.9 Note that the shapes are elements in $\mathcal{O}^{T}$. 


\subsection{Proof that $\alpha^{*}$ is basic}

Since $\alpha^{*}$ has cokernel of order 2, it is enough to prove that there is a set of columns of $\alpha^{*}$ forming a matrix with determinant \pm 2 . Recall that the columns of $\alpha^{*}$ correspond to 1 -cells of $\mathcal{T}$. We shall thus consider graphs in the 1 -skeleton of $\widehat{M}$. We recall some basic results from graph theory. All graphs are assumed to be connected.

Definition 4.10 The incidence matrix of a graph $G$ with vertices $v_{1}, \ldots, v_{k}$ and edges $\varepsilon_{1}, \ldots, \varepsilon_{l}$ is the $k \times l$ matrix $I_{G}$ whose $(i, j)$ entry is 1 if $v_{i}$ is incident to $\varepsilon_{j}$, and 0 otherwise.

Lemma 4.11 The rank of $I_{G}$ is $k-1$. If $G$ is a tree, $I_{G}$ is a $k \times(k-1)$ matrix, and removing any row gives a matrix with determinant \pm 1 .

4.3.1 Case 1: a single cusp In this case the result is trivial. The matrix representation for $\alpha^{*}$ is $(2 \cdots 2)$.

4.3.2 Case 2: multiple cusps, self-edges Suppose $\widehat{M}$ has a self-edge $\varepsilon_{1}$ (an edge joining a cusp to itself), and consider the graph $G$ consisting of the union of $\varepsilon_{1}$ with a maximal tree $T$ (see left image in Figure 7). The columns of $\alpha^{*}$ corresponding to the edges of $G$ then form the matrix

$$
B=\left(\begin{array}{l|l}
2 & \\
0 & I_{T}
\end{array}\right)
$$

which, by Lemma 4.11, has determinant \pm 2 .

4.3.3 Case 3: multiple cusps, no self-edges Pick a face with edges $\varepsilon_{1}, \varepsilon_{2}, \varepsilon_{3}$, and add edges to form a graph $G$ such that $G \backslash \varepsilon_{1}$ is a maximal tree (see right image in Figure 7). The corresponding columns form the matrix

$$
C=I_{G}=\left(\begin{array}{c|c}
1 & \\
0 & I_{T} \\
1 & \\
\hline 0 &
\end{array}\right)
$$

By Lemma $4.11, I_{G}$ is invertible and has determinant \pm 2 . This concludes the proof that $\alpha^{*}$ is basic.

Note that

$$
\operatorname{det}(B)=\operatorname{det}\left(\begin{array}{ll}
2 & 1 \\
& 1
\end{array}\right)=2, \quad \operatorname{det}(C)=\operatorname{det}\left(\begin{array}{lll}
1 & 1 & \\
0 & 1 & 1 \\
1 & & 1
\end{array}\right)=2
$$


ie only the edges and vertices shown in Figure 7 contribute to the determinant.
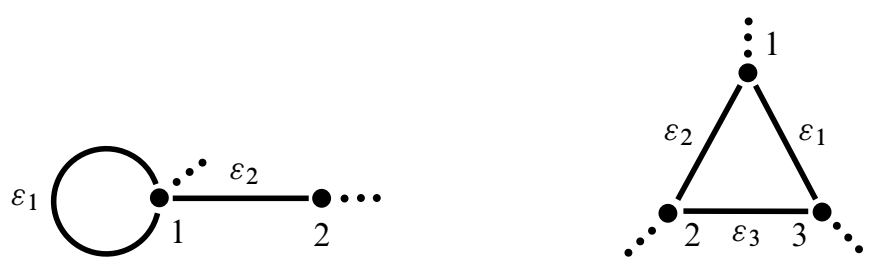

Figure 7: Left: tree $G$ with $1-$ cycle; $G \backslash \varepsilon_{1}$ is a maximal tree. Right: tree $G$ with 3-cycle; $G \backslash \varepsilon_{1}$ is a maximal tree.

Remark 4.12 Trees with 1 - or 3-cycles are also used in [9, Section 4.6] to study index structures.

\section{The Ptolemy field and the trace field}

\subsection{Explicit description of the Ptolemy field}

By Proposition 4.7 any $c \in P_{2}^{\sigma}(\mathcal{T})$ is equivalent to a Ptolemy assignment $c^{\prime}$ whose coordinates for a set of basic generators $\varepsilon_{i_{1}}, \ldots, \varepsilon_{i_{v}}$ is 1 . In particular, it follows that the Ptolemy field (see Definition 1.13) of $c \in P_{2}^{\sigma}(\mathcal{T})$ is given by

$$
k_{c}=k_{c^{\prime}}=\mathbb{Q}\left(\left\{c_{\varepsilon_{1}}^{\prime}, \ldots, c_{\varepsilon_{e}}^{\prime}\right\}\right) .
$$

Definition 5.1 Let $\rho: \pi_{1}(M) \rightarrow \operatorname{PSL}(2, \mathbb{C})$ be a representation. The trace field of $\rho$ is the field generated by the traces of elements in the image. We denote it $k_{\rho}$.

Our main result is the following. We defer the proof to Section 5.4.

Theorem 5.2 Let $c \in P_{2}^{\sigma}(\mathcal{T})_{\text {red }}$. If the corresponding generic representation $\rho$ of $\pi_{1}(M)$ in $\operatorname{PSL}(2, \mathbb{C})$ is boundary-nontrivial, the Ptolemy field of $c$ equals the trace field of $\rho$.

Remark 5.3 Note that if $c \in P_{2}^{\sigma}(\mathcal{T})_{\text {red }}$ is in a degree- 0 component, the Ptolemy field is a number field. 


\subsection{The setup of the proof}

Since the natural cocycle is given in terms of the Ptolemy coordinates, it follows that $\rho$ is defined over the Ptolemy field. Hence, the trace field is a subfield of the Ptolemy field.

Fix a maximal tree $G$ with $1-$ or 3-cycle as in Figure 7. As explained in Section 4.3, the edges of $G$ are basic generators of $\alpha^{*}$. We may thus assume without loss of generality that the Ptolemy coordinates $c_{i}$ of the edges $\varepsilon_{i}$ of $G$ are 1. By (5-1), it is thus enough to show that the Ptolemy coordinates of the remaining 1-cells are in the trace field.

Let $\gamma$ denote the (lifted) natural cocycle of $c$. Then $\gamma$ assigns to each edge path $p$ in $\widehat{M}$ a matrix $\gamma(p) \in \operatorname{SL}(2, \mathbb{C})$. Let

$$
\alpha(a)=\left(\begin{array}{cc}
0 & -a^{-1} \\
a & 0
\end{array}\right), \quad \beta(b)=\left(\begin{array}{ll}
1 & b \\
0 & 1
\end{array}\right)
$$

As shown in Figure 2, $\gamma$ takes long and short edges to elements of the form $\alpha(a)$ and $\beta(b)$, respectively, where $a$ and $b$ are given in terms of the Ptolemy coordinates.

Since $\rho$ is boundary-nontrivial there exists, for each cusp $i$ of $M$, a peripheral loop $M_{i}$ with $\gamma\left(M_{i}\right) \in P$ nontrivial. We shall here refer to such loops as nontrivial. Fix such nontrivial loops $M_{i}$, once and for all, and let $m_{i} \neq 0$ be such that $\gamma\left(M_{i}\right)=\beta\left(m_{i}\right)$. For any edge path $p$ with endpoint on a cusp $i$ we can alter $M_{i}$ by a conjugation if necessary (this does not change $m_{i}$ ) so that $p$ is composable with $M_{i}$.

\subsection{Proof for one cusp}

We first prove Theorem 5.2 in the case where there is only one cusp. In this case, all edges are self-edges, and $T$ consists of a single edge $\varepsilon_{1}$.

Lemma 5.4 For any self-edge $\varepsilon$, we have $m_{1} c_{\varepsilon} \in k_{\rho}$.

Proof Let $X_{1}$ be a peripheral path such that $X_{1} \varepsilon$ is a loop (see the left image in Figure 8), and let $x_{1}$ be such that $\gamma\left(X_{1}\right)=\beta\left(x_{1}\right)$. We have

$$
\operatorname{Tr}\left(\gamma\left(X_{1} \varepsilon\right)\right)=\operatorname{Tr}\left(\beta\left(x_{1}\right) \alpha\left(c_{\varepsilon}\right)\right)=\operatorname{Tr}\left(\left(\begin{array}{cc}
1 & x_{1} \\
0 & 1
\end{array}\right)\left(\begin{array}{cc}
0 & -c_{\varepsilon}^{-1} \\
c_{\varepsilon} & 0
\end{array}\right)\right)=x_{1} c_{\varepsilon} \in k_{\rho} .
$$

Applying the same computation to the loop $X_{1} M_{1} \varepsilon$ yields

$$
\operatorname{Tr}\left(\beta\left(x_{1}\right) \beta\left(m_{1}\right) \alpha\left(c_{\varepsilon}\right)\right)=\left(x_{1}+m_{1}\right) c_{\varepsilon} \in k_{\rho},
$$

and the result follows. 
Since the Ptolemy coordinate of $\varepsilon_{1}$ is 1 , it follows that $m_{1} \in k_{\rho}$. Since all edges are self-edges, we have $c_{\varepsilon} \in k_{\rho}$ for all 1 -cells $\varepsilon$. This concludes the proof in the one-cusped case.
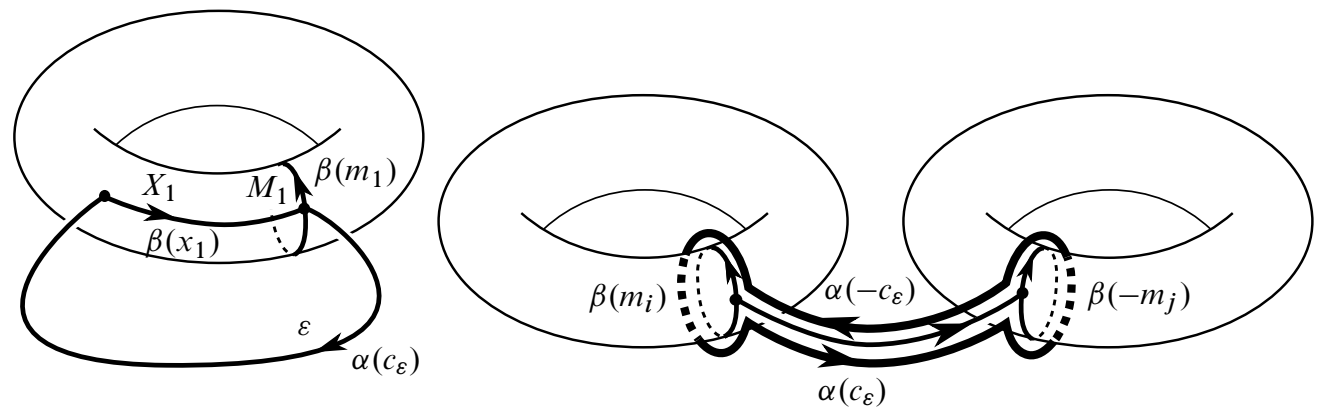

Figure 8: Left: self-edge. Right: edge between cusps.

\subsection{The general case}

The general case follows the same strategy, but is more complicated since it involves edge paths between multiple cusps.

Lemma 5.5 If $\varepsilon$ is a self-edge from cusp $i$ to itself, $m_{i} c_{\varepsilon} \in k_{\rho}$.

Proof The proof is identical to that of Lemma 5.4.

Lemma 5.6 If two (distinct) cusps $i$ and $j$ are joined by an edge $\varepsilon$ in $G$, we have

$$
m_{i} m_{j} \in k_{\rho}
$$

Proof Consider the loop $\varepsilon_{j} \bar{M}_{j} \bar{\varepsilon}_{j} M_{i}$ shown in the right image in Figure 8. A simple computation shows that

$$
\operatorname{Tr}\left(\alpha\left(c_{\varepsilon}\right) \beta\left(-m_{j}\right) \alpha\left(-c_{\varepsilon}\right) \beta\left(m_{i}\right)\right)=2+m_{i} m_{j} c_{\varepsilon}^{2} .
$$

Since $\varepsilon \in T, c_{\varepsilon}=1$, and the result follows.

More generally, the following holds.

Lemma 5.7 We have $m_{i} \in k_{\rho}$ for all cusps $i$.

Proof If $G$ is a tree with 1 -cycle, then $c_{1}=1$, so Lemma 5.5 implies that $m_{1} \in k_{\rho}$. Inductively applying Lemma 5.6 for the edge $\varepsilon_{j}$ connecting cusp $i=j-1$ and $j$ 
implies the result. If $G$ is a tree with 3 -cycle, the Ptolemy coordinates $c_{1}, c_{2}$ and $c_{3}$ are 1 , so the edges of the face are labeled by $\alpha(1)$ and $\beta(-1)$ only (see Figure 2 ). Inserting the peripheral loops $M_{i}$ as in Figure 9, we obtain

$$
\operatorname{Tr}\left(\beta(-1) \beta\left(m_{1}\right) \alpha(1) \beta(-1) \beta\left(m_{2}\right) \alpha(1) \beta(-1) \beta\left(m_{3}\right) \alpha(1)\right) \in k_{\rho} .
$$

By an elementary computation, the trace equals

$$
m_{1} m_{2} m_{3}-m_{1} m_{2}-m_{2} m_{3}-m_{3} m_{1}+2 \in k_{\rho} .
$$

By Lemma 5.6, $m_{i} m_{j} \in k_{\rho}$, so $m_{1} \in k_{\rho}$. The result now follows as above by inductively applying Lemma 5.6.

Let $\varepsilon$ be an arbitrary 1-cell. If $\varepsilon$ is a self-edge, Lemmas 5.5 and 5.7 imply that $c_{\varepsilon} \in k_{\rho}$. Otherwise, there exists an edge path $p$ in the maximal tree $G \backslash \varepsilon_{1}$ such that $p * \varepsilon$ is a loop in $\widehat{M}$. By relabeling the cusps and edges if necessary, we may assume that $p=\varepsilon_{i+1} * \varepsilon_{i+2} * \cdots * \varepsilon_{j}$, where $\varepsilon_{k}$ goes from cusp $k-1$ to cusp $k$. Pick peripheral paths $X_{k}$ on cusp $k$ connecting the ends (in $M$, not $\widehat{M}$ ) of edges $\varepsilon_{k}$ and $\varepsilon_{k+1}$ (see Figure 10). We obtain a loop that can be composed with arbitrary powers of the peripheral loops $M_{i}, \ldots, M_{j}$. We thus obtain the following traces (where $b_{k} \in \mathbb{Z}$ ):

$$
\begin{aligned}
& \operatorname{Tr}\left(\beta\left(x_{i}+b_{i} m_{i}\right) \alpha\left(c_{i+1}\right) \beta\left(x_{i+1}+b_{i+1} m_{i+1}\right) \alpha(\right.\left.c_{i+2}\right) \\
& \\
&\left.\cdot \beta\left(x_{j}+b_{j} m_{j}\right) \alpha\left(c_{\varepsilon}\right)\right) \in k_{\rho} .
\end{aligned}
$$

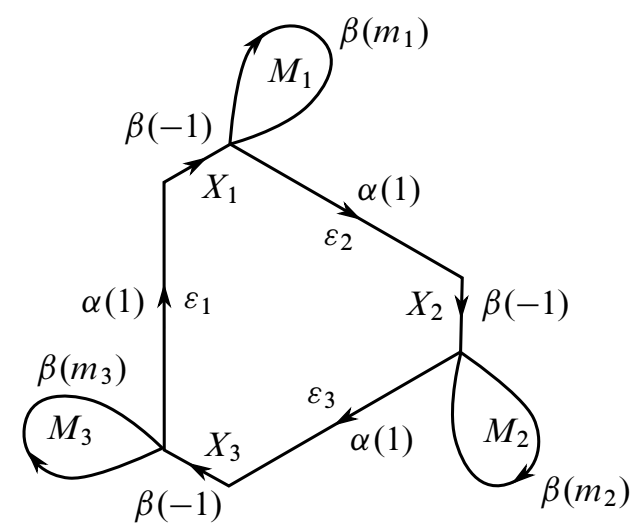

Figure 9: 3-cycle case

It will be convenient to regard $\operatorname{Tr}\left(\beta\left(x_{i}\right) \alpha\left(c_{i+1}\right) \beta\left(x_{i+1}\right) \alpha\left(c_{i+2}\right) \cdots \beta\left(x_{j}\right) \alpha\left(c_{\varepsilon}\right)\right)$ as a function of variables $x_{i}$ (disregarding that the $x_{i}$ are fixed expressions of the Ptolemy coordinates). 


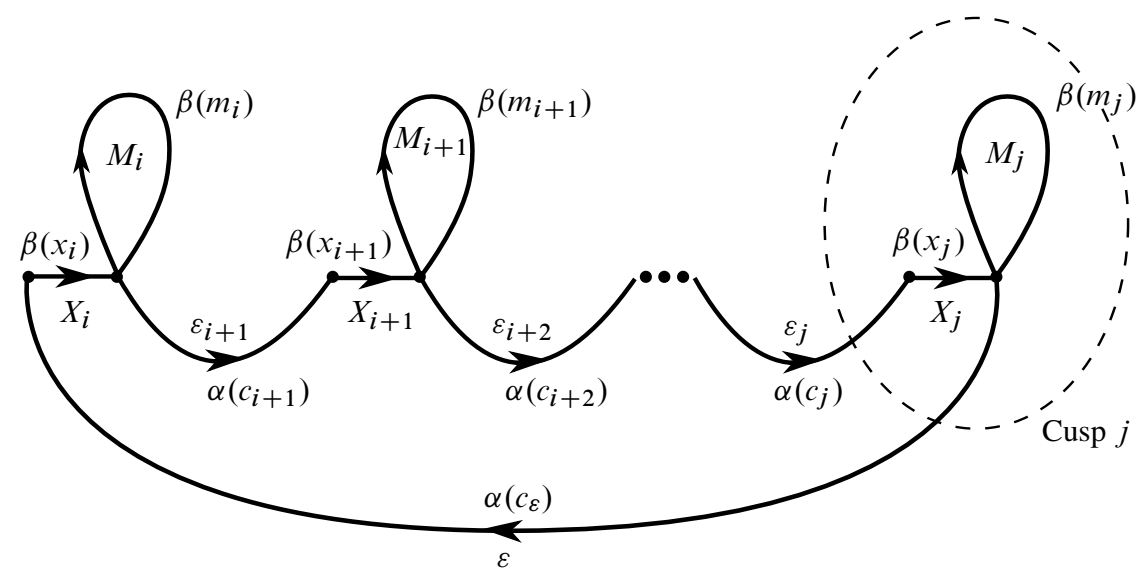

Figure 10: Arbitrary edge $\varepsilon$

Definition 5.8 Given a function $f\left(x_{1}, \ldots, x_{r}\right)$, let $\Delta_{i} f$ be the function given by

$$
\Delta_{i} f(h)=f\left(x_{1}, \ldots, x_{i}+h, \ldots, x_{r}\right)-f\left(x_{1}, \ldots, x_{i}, \ldots, x_{r}\right) .
$$

The following is elementary.

Lemma 5.9 If $f\left(x_{1}, \ldots, x_{r}\right)$ is a polynomial where the exponents of all variables $x_{i}$ are 0 or 1 , and where the highest-degree term is $a x_{1} x_{2} \cdots x_{r}$, we have

$$
\Delta_{r}\left(\cdots \Delta_{2}\left(\Delta_{1} f\left(h_{1}\right)\right)\left(h_{2}\right) \cdots\right)=a h_{1} h_{2} \cdots h_{r},
$$

and the left-hand side is thus independent of the $x_{i}$.

If, for example, $f\left(x_{1}, x_{2}\right)=x_{1} x_{2}$, we have

$$
\begin{aligned}
\Delta_{1} f\left(h_{1}\right) & =\left(x_{1}+h_{1}\right) x_{2}-x_{1} x_{2}=h_{1} x_{2}, \\
\Delta_{2}\left(\Delta_{1} f\left(h_{1}\right)\right)\left(h_{2}\right) & =h_{1}\left(x_{2}+h_{2}\right)-h_{1} x_{2}=h_{1} h_{2} .
\end{aligned}
$$

Lemma 5.10 Let $x_{1}, \ldots, x_{r}$ be variables and $y_{1}, \ldots, y_{r}$ be constants. The expression

$$
\operatorname{Tr}\left(\beta\left(x_{1}\right) \alpha\left(y_{1}\right) \cdots \beta\left(x_{r}\right) \alpha\left(y_{r}\right)\right)
$$

is a polynomial in the $x_{i}$ whose unique highest-degree term is $\prod_{i=1}^{r} y_{i} \prod_{i=1}^{r} x_{i}$. Moreover, for each monomial term, the exponent of each variable is either 1 or 0 .

Proof This follows by induction on $r$. 
Applying Lemmas 5.10 and 5.9 to the function

$$
f\left(x_{i}, \ldots, x_{j}\right)=\operatorname{Tr}\left(\beta\left(x_{i}\right) \alpha\left(c_{i+1}\right) \beta\left(x_{i+1}\right) \alpha\left(c_{i+2}\right) \cdots \beta\left(x_{j}\right) \alpha\left(c_{\varepsilon}\right)\right),
$$

we obtain

$$
\left(m_{i} m_{i+1} \cdots m_{j} c_{i} c_{i+1} \cdots c_{j}\right) c_{\varepsilon} \in k_{\rho} .
$$

Since all $m_{i}$ are in $k_{\rho}$ by Lemma 5.7, and all $c_{i}$ are 1 (since $\varepsilon_{i} \in T$ ), it follows that $c_{\varepsilon}$ is in $k_{\rho}$. This concludes the proof.

\subsection{Proof of Proposition 1.10}

The fact that $\mathcal{R}$ factors follows from the fact that the diagonal action only changes the decoration (by diagonal elements; see Remark 4.1), not the representation. Since the preimage of the right map in (1-4) is parametrized by choices of lifts, ie elements in $Z^{1}(\widehat{M} ; \mathbb{Z} / 2 \mathbb{Z})$, all that remains is to show that the only freedom in the choice of decoration of a boundary-nontrivial representation is the diagonal action. This follows from results in [10]: a decoration is an equivariant map

$$
D: \widehat{\widetilde{M}}^{(0)} \rightarrow \operatorname{PSL}(2, \mathbb{C}) / P,
$$

and is thus determined by its image of lifts $\tilde{e}_{1}, \ldots, \widetilde{e}_{v}$ of the cusps of $M$. The freedom in the choice of $D\left(\widetilde{e}_{i}\right)$ is the choice of a coset $g P$ satisfying $g \rho\left(\operatorname{Stab}\left(\widetilde{e}_{i}\right)\right) g^{-1} \subset P$, where $\operatorname{Stab}\left(\widetilde{e}_{i}\right) \subset \pi_{1}(M)$ is the stabilizer of $\widetilde{e}_{i}$, ie a peripheral subgroup corresponding to cusp $i$. Hence, if $\rho\left(\operatorname{Stab}\left(\widetilde{e}_{i}\right)\right)$ is nontrivial, the freedom is right-multiplication by a diagonal matrix (if it is trivial, any coset works). Hence, if $\rho$ is boundary-nontrivial, the only freedom in choosing a decoration is the diagonal action.

\section{Ptolemy varieties for $n>2$}

Many of our results generalize in a straightforward way to the higher-rank Ptolemy varieties $P_{n}(\mathcal{T})$. We recall the definition of these below, and refer to [10; 8] for details. We identify all simplices of $\mathcal{T}$ with a standard simplex

$$
\Delta_{n}^{3}=\left\{\left(x_{0}, x_{1}, x_{2}, x_{3}\right) \in \mathbb{R}^{4} \mid 0 \leq x_{i} \leq n, x_{0}+x_{1}+x_{2}+x_{3}=n\right\}
$$

and regard $\widehat{M}$ as a quotient of a disjoint union $\bigsqcup_{k=1}^{s} \Delta_{n, k}^{3}$, with a copy $\Delta_{n, k}^{3}$ of $\Delta_{n}^{3}$ for each simplex $k$ of $\mathcal{T}$. Define

$$
\Delta_{n}^{3}(\mathbb{Z})=\Delta_{n}^{3} \cap \mathbb{Z}^{4},
$$

and define $\dot{\Delta}_{n}^{3}(\mathbb{Z})$ to be $\Delta_{n}^{3}(\mathbb{Z})$ with the four vertex points removed. A point in $M$ in the image of $\bigsqcup_{k=1}^{S} \dot{\Delta}_{n, k}^{3}(\mathbb{Z})$ is called an integral point of $M$. 


\subsection{Definition of the Ptolemy variety}

Assign to each $(t, k) \in \Delta_{n, k}^{3}(\mathbb{Z})$ a Ptolemy coordinate $c_{t, k}$. For each simplex $k$, we have $\left|\Delta_{n-2}(\mathbb{Z})\right|=\left(\begin{array}{c}n+1 \\ 3\end{array}\right)$ Ptolemy relations

$$
\begin{aligned}
& c_{\alpha+1001, k} c_{\alpha+0110, k}+c_{\alpha+1100, k} c_{\alpha+} \\
& \quad=c_{\alpha+1011, k} c_{\alpha+0101, k}, \quad \alpha \in \Delta_{n-2}(\mathbb{Z}),
\end{aligned}
$$

as well as identification relations

$$
c_{t, k}= \pm c_{t^{\prime}, k^{\prime}} \quad \text { when }(t, k) \sim\left(t^{\prime}, k^{\prime}\right) .
$$

Remark 6.1 The signs in (6-3) depend in a nontrivial way on the face pairings (see [8]). For ordered triangulations the signs are always positive. As in Remark 3.3 we can eliminate the identification relations by selecting a representative of each integral point of $M$.

Definition 6.2 The Ptolemy variety $P_{n}(\mathcal{T})$ is the subset of the affine algebraic set defined by the Ptolemy and identification relations, consisting of the points where all Ptolemy coordinates are non-zero.

For general $n$ we denote the group of upper-triangular matrices with 1 on the diagonal by $N$ (instead of $P$ ). As in (1-2) we have

(6-4) $\left\{\right.$ Points in $\left.P_{n}(\mathcal{T})\right\} \stackrel{1-1}{\longleftrightarrow}\{$ Natural $(\operatorname{SL}(n, \mathbb{C}), N)$-cocycles on $M\}$

$\stackrel{1-1}{\longleftrightarrow}\{$ Generically decorated $(\operatorname{SL}(n, \mathbb{C}), N)$-representations $\}$.

\subsection{The diagonal action}

Let $D$ be the group of diagonal matrices in $\operatorname{SL}(n, \mathbb{C})$. We identify $D$ with the torus $\left(\mathbb{C}^{*}\right)^{n-1}$ via the identification

$(6-5)\left(\mathbb{C}^{*}\right)^{n-1} \rightarrow D, \quad\left(a_{1}, \ldots, a_{n-1}\right) \mapsto \operatorname{diag}\left(a_{1}, a_{2} / a_{1}, \ldots, a_{n-1} / a_{n-2}, 1 / a_{n-1}\right)$.

As in Remark 4.1, we have a diagonal action of the torus $T=D^{v}$ on the set of decorated representations, where $\left(D_{1}, \ldots, D_{v}\right) \in T$ acts by replacing the coset $g N$ assigned to a vertex $w$ by $g D_{i} N$, where $i$ is the cusp corresponding to $w$. The corresponding action on $P_{n}(\mathcal{T})$ is described in Lemma 6.4 below.

Let $C_{1}^{n}$ be the group generated by the integral points of $M$, and let $C_{0}^{n}=C_{0} \otimes \mathbb{Z}^{n-1}$. In Garoufalidis and Zickert [11] we defined maps

$$
\alpha: C_{0}^{n} \rightarrow C_{1}^{n}, \quad \alpha^{*}: C_{1}^{n} \rightarrow C_{0}^{n},
$$


generalizing (4-2). The map $\alpha^{*}$ takes an integral point $(t, k)$ to $\sum x_{i} \otimes e_{t_{i}}$, where $x_{i}$ is the cusp determined by vertex $i$ of simplex $k$. We shall not need the definition of $\alpha$.

Lemma 6.3 [11] The map $\alpha^{*}$ is surjective with cokernel $\mathbb{Z} / n \mathbb{Z}$.

By selecting an ordering of the natural generators of $C_{0}^{n}$ and $C_{1}^{n}$, we regard $\alpha$ and $\alpha^{*}$ as matrices. The following is an elementary consequence of (6-4).

Lemma 6.4 The diagonal action of $T=\left(\mathbb{C}^{*}\right)^{v(n-1)}$ on $P_{n}(\mathcal{T})$ and the corresponding action on the coordinate ring $\mathcal{O}$ of $P_{n}(\mathcal{T})$ are given, respectively, by

$$
(x c)_{t}=\left(\prod_{j=1}^{v(n-1)} x_{j}^{\alpha_{t j}}\right) c_{t}, \quad x\left(c^{w}\right)=\prod_{j=1}^{v(n-1)} x_{j}^{\alpha^{*}(w)_{j}} c^{w} .
$$

Corollary 6.5 The ring of invariants $\mathcal{O}^{T}$ is generated by $c^{w_{1}}, \ldots, c^{w_{r}}$, where $r=$ $\operatorname{rank}\left(C_{1}^{n}\right)-\operatorname{rank}\left(C_{0}^{n}\right)$ and $w_{1}, \ldots, w_{r}$ are a basis for $\operatorname{Ker} \alpha^{*}$.

Definition 6.6 The Ptolemy field of a Ptolemy assignment $c \in P_{n}(\mathcal{T})$ is defined as

$$
k_{c}=\mathbb{Q}\left(c^{w_{1}}, \ldots, c^{w_{r}}\right),
$$

where $w_{1}, \ldots, w_{r}$ are (integral) generators of $\operatorname{Ker} \alpha^{*}$.

The following is proved in Section 6.4.

Proposition 6.7 The map $\alpha^{*}: C_{1}^{n} \rightarrow C_{0}^{n}$ is basic.

Corollary 6.8 Let $p_{1}, \ldots, p_{(n-1) v}$ be integral points that are basic generators of $C_{1}^{n}$. The ring $\mathcal{O}^{T}$ is generated by the Ptolemy relations together with the relations $c_{p_{1}}=$ $\cdots=c_{p_{(n-1) v}}=1$. Equivalently, the reduced Ptolemy variety is isomorphic to the subvariety of $P_{n}(\mathcal{T})$ consisting of Ptolemy assignments with $c_{p_{i}}=1$.

Proof This follows the proof of Proposition 4.7 word by word.

Remark 6.9 This is how the Ptolemy varieties and Ptolemy fields at [5] are computed. 


\subsection{Representations}

Definition 6.10 Let $\rho$ be an $(\operatorname{SL}(n, \mathbb{C}), N)$-representation, and let $I_{i}$ denote the image of the peripheral subgroup corresponding to cusp $i$. We say that $\rho$ is boundarynon-degenerate if each $I_{i}$ has an element whose Jordan canonical form has a single (maximal) Jordan block.

\section{Proposition 6.11 The map}

$$
\mathcal{R}: P_{n}(\mathcal{T})_{\text {red }} \rightarrow\{(\operatorname{SL}(n, \mathbb{C}), N) \text {-representations }\} / \text { Conj }
$$

maps onto the generic representations, and the preimage of a generic boundary-nondegenerate representation consists of a single point.

Proof The proof is identical to the proof in Section 5.5 for $n=2$.

Conjecture 6.12 The Ptolemy field of a generic, boundary-non-degenerate representation is equal to its trace field.

Remark 6.13 Much of the theory also works for $\operatorname{PSL}(n, \mathbb{C})$-representations by means of obstruction classes in $H^{2}(\widehat{M} ; \mathbb{Z} / n \mathbb{Z})$. When $n$ is even, obstruction classes in $H^{2}(\widehat{M} ; \mathbb{Z} / 2 \mathbb{Z})$ were defined in $[10]$ for representations in $p \operatorname{SL}(n, \mathbb{C})=\operatorname{SL}(n, \mathbb{C}) / \pm I$. For $\operatorname{PSL}(n, \mathbb{C})$-representations, both the Ptolemy field and the trace field are only defined up to $n^{\text {th }}$ roots of unity. The generalized obstruction classes are used on the website [5] and will be explained in a forthcoming publication.

\subsection{Proof that $\alpha^{*}$ is basic}

By Lemma 6.3, we need to prove the existence of integral points such that the corresponding columns of $\alpha^{*}$ form a matrix of determinant $\pm n$. As in Section 4.3 we split the proof into three cases.

6.4.1 Basic matrix algebra Let $I_{k}$ be the identity matrix, $R_{k}$ the sparse matrix whose first row contains entirely of 1 's, $S_{k}$ the sparse matrix whose lower diagonal consists of 1 's $\left(S_{1}=0\right)$, and $T_{k}$ the sparse matrix whose lower right entry is 1 . The index $k$ denotes that the matrices are $k \times k$. For $k=3$, we have

$$
R_{3}=\left(\begin{array}{lll}
1 & 1 & 1 \\
& & \\
& &
\end{array}\right), \quad S_{3}=\left(\begin{array}{ll}
1 & \\
& 1
\end{array}\right), \quad T_{3}=\left(\begin{array}{l} 
\\
\\
\end{array}\right) \text {. }
$$

Lemma 6.14 We have

$$
\operatorname{det}\left(I_{k}+R_{k}-S_{k}\right)=k+1, \quad \operatorname{det}\left(I_{k}+R_{k}+T_{k}-S_{k}\right)=2 k+1 .
$$


Proof This follows, for example, by expanding the determinant using the last column. The matrices $I_{k}+R_{k}-S_{k}$ are shown below for $k=1,2,3$ and 4:

$$
\text { (2), }\left(\begin{array}{rr}
2 & 1 \\
-1 & 1
\end{array}\right), \quad\left(\begin{array}{rrr}
2 & 1 & 1 \\
-1 & 1 & \\
& -1 & 1
\end{array}\right), \quad\left(\begin{array}{rrrr}
2 & 1 & 1 & 1 \\
-1 & 1 & & \\
& -1 & 1 & \\
& & -1 & 1
\end{array}\right) \text {. }
$$

For $I_{k}+R_{k}+T_{k}-S_{k}$, the only difference is that the lower right entry is now 2 .

Lemma 6.15 Let $A, B, C, D$ be $k \times k, k \times l, l \times k$, and $l \times l$ matrices, respectively, and let $M=\left(\begin{array}{cc}A & B \\ C & D\end{array}\right)$. If $D$ is invertible, we have

$$
\operatorname{det}(M)=\operatorname{det}(D) \operatorname{det}\left(A-B D^{-1} C\right) .
$$

Proof This follows from the identity

$$
\left(\begin{array}{ll}
A & B \\
C & D
\end{array}\right)=\left(\begin{array}{ll}
I & B \\
0 & D
\end{array}\right)\left(\begin{array}{cc}
A-B D^{-1} C & 0 \\
D^{-1} C & I
\end{array}\right) .
$$

6.4.2 One cusp Pick any face of $\mathcal{T}$ and consider the integral points shown in Figure 11. Let $A_{n}$ be the $(n-1) \times(n-1)$ matrix formed by the corresponding columns of $\alpha^{*}$. The columns are ordered as shown in the figures, and the rows, ie the generators $x \otimes e_{i}$ of $C_{0}^{n}$, are ordered in the natural way (increasing in $i$ ). The following is an immediate consequence of the definition of $\alpha^{*}$.
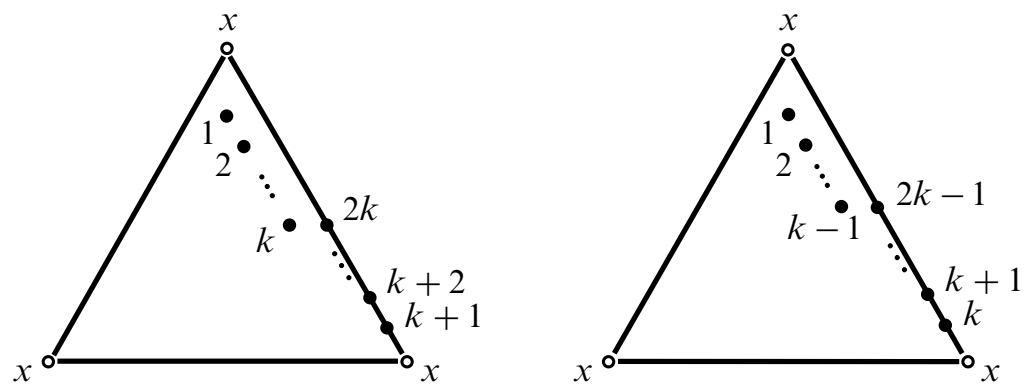

Figure 11: Left: basic generators, $n=2 k+1$. Right: basic generators, $n=2 k$.

Lemma 6.16 The matrix $A_{n}$ is given by

$$
A_{2 k+1}=\left(\begin{array}{cc}
I_{k}+R_{k}+T_{k} & I_{k} \\
S_{k} & I_{k}
\end{array}\right), \quad A_{2 k}=\left(\begin{array}{ccc}
2 & 0 \cdots 01 & 0 \\
0 & I_{k-1}+R_{k-1} & I_{k-1} \\
0 & S_{k-1} & I_{k-1}
\end{array}\right) \text {. }
$$


Corollary 6.17 The determinant of $A_{n}$ is $\pm n$.

Proof This follows from Lemma 6.15 and Lemma 6.14.

6.4.3 Multiple cusps, self-edges Pick a face with a self-edge, and extend to a maximal tree with 1-cycle $G$ as in the left image in Figure 7. Let $T=G \backslash \varepsilon_{1}$, and let $B_{n}$ denote the matrix formed by the columns of $\alpha^{*}$ corresponding to the face points shown in the left image in Figure 12 together with the edge points on $T$. We order the generators $x_{i} \otimes e_{j}$ of $C_{0}^{n}$ as

$$
x_{1} \otimes e_{1}, \ldots, x_{1} \otimes e_{n-1}, \quad x_{2} \otimes e_{n-1}, \ldots, x_{2} \otimes e_{1},
$$

with a similar scheme for the other vertices. The following is an immediate consequence of the definition of $\alpha^{*}$.

Lemma 6.18 The matrix $B_{n}$ is given by

$$
B_{n}=\left(\begin{array}{c|c}
I_{n-1}+R_{n-1} & \\
S_{n-1} & I_{T} \otimes \mathbb{Z}^{n-1} \\
\hline 0 &
\end{array}\right)
$$

where $I_{T} \otimes \mathbb{Z}^{n-1}$ is the matrix obtained from $I_{T}$ by replacing each non-zero entry by $I_{n-1}$.

Corollary 6.19 The determinant of $B_{n}$ is $\pm n$.

Proof This follows from

$$
\operatorname{det}\left(B_{n}\right)= \pm \operatorname{det}\left(\begin{array}{cc}
I_{n-1}+R_{n-1} & I_{n-1} \\
S_{n-1} & I_{n-1}
\end{array}\right)= \pm n,
$$

where the second equality follows from Lemmas 6.15 and 6.14 .

6.4.4 Multiple cusps, no self-edge Pick a maximal tree with 3-cycle $G$, and let $C_{n}$ be the matrix formed by the columns of $\alpha^{*}$ corresponding to the face points in the right image in Figure 12 together with the edge points on $T=G \backslash \varepsilon_{1}$.

Lemma 6.20 The matrix $C_{n}$ is given by

$$
C_{n}=\left(\begin{array}{c|c}
I_{n-1} \\
S_{n-1} \\
R_{n-1} \\
\hline 0
\end{array} \mid I_{T} \otimes \mathbb{Z}^{n-1}\right) .
$$



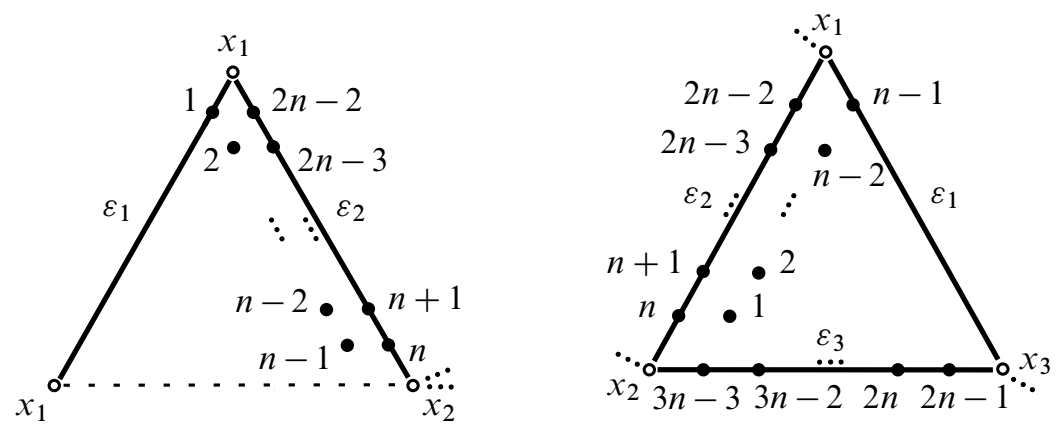

Figure 12: Left: basic generators, tree with 1-cycle. Right: basic generators, tree with 3-cycle.

Corollary 6.21 The determinant of $C_{n}$ is $\pm n$.

Proof We have

$$
\operatorname{det}\left(C_{n}\right)= \pm \operatorname{det}(M), \quad M=\left(\begin{array}{lll}
I_{n-1} & I_{n-1} & \\
S_{n-1} & I_{n-1} & I_{n-1} \\
R_{n-1} & & I_{n-1}
\end{array}\right) .
$$

Using Lemma 6.15 with

$$
A=\left(\begin{array}{cc}
I_{n-1} & I_{n-1} \\
S_{n-1} & I_{n-1}
\end{array}\right), \quad B=\left(\begin{array}{c}
0 \\
I_{n-1}
\end{array}\right), \quad C=\left(\begin{array}{ll}
R_{n-1} & 0
\end{array}\right), \quad D=I_{n-1},
$$

we have

(6-20) $\quad \operatorname{det}(M)=\operatorname{det}\left(\begin{array}{cc}I_{n-1} & I_{n-1} \\ S_{n-1}-R_{n-1} & I_{n-1}\end{array}\right)=\operatorname{det}\left(I_{n-1}+R_{n-1}-S_{n-1}\right)=n$;

the second equation follows from Lemma 6.15 and the third from Lemma 6.14.

This concludes the proof that $\alpha^{*}$ is basic.

\section{References}

[1] W Bosma, J Cannon, C Playoust, The Magma algebra system, I: The user language, J. Symbolic Comput. 24 (1997) 235-265

[2] D Calegari, Real places and torus bundles, Geom. Dedicata 118 (2006) 209-227 MR2239457

[3] M Culler, N M Dunfield, J R Weeks, SnapPy: A computer program for studying the geometry and topology of 3-manifolds http://snappy.computop.org/

[4] N M Dunfield, Cyclic surgery, degrees of maps of character curves, and volume rigidity for hyperbolic manifolds, Invent. Math. 136 (1999) 623-657 MR1695208 
[5] E Falbel, S Garoufalidis, A Guilloux, M Görner, P-V Koseleff, F Rouillier, C Zickert, CURVE database http://curve.unhyperbolic.org/database.html

[6] E Falbel, P V Koseleff, F Rouillier, Representations of fundamental groups of 3manifolds into $\mathrm{PGL}(3, \mathbb{C})$ : Exact computations in low complexity arXiv:1307.6697

[7] V Fock, A Goncharov, Moduli spaces of local systems and higher Teichmüller theory, Publ. Math. Inst. Hautes Études Sci. (2006) 1-211 MR2233852

[8] S Garoufalidis, M Goerner, C K Zickert, Gluing equations for PGL(n, $\mathbb{C})$-representations of 3-manifolds arXiv:1207.6711 To appear in Alg. \& Geom. Topol.

[9] S Garoufalidis, C D Hodgson, H Rubinstein, H Segerman, 1-efficient triangulations and the index of a cusped hyperbolic 3-manifold arXiv:1303.5278 To appear in Geom. \& Topol.

[10] S Garoufalidis, D P Thurston, CK Zickert, The complex volume of SL(n, $\mathbb{C})-$ representations of 3-manifolds arXiv:1111.2828 To appear in Duke Math. J.

[11] S Garoufalidis, C K Zickert, The symplectic properties of the PGL(n, C)-gluing equations arXiv:1310.2497 To appear in J. Quantum Topol.

[12] C Maclachlan, A W Reid, The arithmetic of hyperbolic 3-manifolds, Graduate Texts in Mathematics 219, Springer, New York (2003) MR1937957

[13] W D Neumann, Combinatorics of triangulations and the Chern-Simons invariant for hyperbolic 3-manifolds, from: “Topology '90”, Ohio State Univ. Math. Res. Inst. Publ. 1, de Gruyter, Berlin (1992) 243-271 MR1184415

[14] C K Zickert, The volume and Chern-Simons invariant of a representation, Duke Math. J. 150 (2009) 489-532

School of Mathematics, Georgia Institute of Technology

686 Cherry Street, Atlanta, GA 30332-0160, USA

Pixar Animation Studios

1200 Park Avenue, Emeryville, CA 94608, USA

Department of Mathematics, University of Maryland

College Park, MD 20742-4015, United States

stavros@math.gatech.edu, enischte@gmail.com, zickert@umd.edu

http://www.math.gatech.edu/ stavros, http://www.unhyperbolic.org/, http: //www2 . math. umd. edu/ zickert

Received: 21 January $2014 \quad$ Revised: 9 May 2014 
